\title{
Further structural and functional properties of mini-lipoxygenase, an active fragment of soybean lipoxygenase-1
}

\author{
Mauro Maccarrone ${ }^{\mathrm{a}, 1, *}$, Almerinda Di Venere ${ }^{\mathrm{b}, \mathrm{c}, 1}$, Guus van Zadelhoff $^{\mathrm{d}}$, Giampiero Mei ${ }^{\mathrm{b}, \mathrm{c}}$, \\ Gerrit Veldink $^{\mathrm{d}}$, Nicola Rosato ${ }^{\mathrm{b}, \mathrm{c}}$ and Alessandro Finazzi-Agrò ${ }^{\mathrm{b}}$ \\ ${ }^{a}$ Department of Biomedical Sciences, University of Teramo, Teramo, Italy \\ ${ }^{\mathrm{b}}$ Department of Experimental Medicine and Biochemical Sciences, University of Rome "Tor Vergata", \\ Rome, Italy \\ ${ }^{\mathrm{c}}$ Istituto Nazionale di Fisica della Materia, Genova, Italy \\ ${ }^{\mathrm{d}}$ Bijvoet Center for Biomolecular Research, Department of Bio-organic Chemistry, Utrecht University, \\ Utrecht, The Netherlands
}

\begin{abstract}
Lipoxygenases (Loxs) form a homologous family of non-heme, non-sulfur iron containing lipid-peroxidizing enzymes, which catalyze the dioxygenation of polyunsaturated fatty acids to the corresponding hydroperoxy derivatives. Soybean lipoxygenase-1 (Lox-1) is widely used as a prototype for studying the structural and functional properties of lipoxygenases. Tryptic digestion of soybean Lox-1 is known to produce a $60 \mathrm{kDa}$ fragment, termed "mini-Lox", which shows enhanced catalytic efficiency and higher membrane binding ability than the native enzyme (M. Maccarrone, M.L. Salucci, G. van Zadelhoff, F. Malatesta, G. Veldink, J.F.G. Vliegenthart and A. Finazzi-Agrò, Biochemistry 40 (2001), 6819-6827). In this study, we have investigated the stability of mini-Lox in guanidinium hydrochloride $(\mathrm{GdHCl})$ and under high pressure by fluorescence and circular dichroism spectroscopy. The denaturation experiments demonstrate that mini-Lox is a rather unstable molecule, which undergoes a two-step unfolding transition. Both chemical- and physical-induced denaturation suggest that mini-Lox is more hydrated than Lox-1, an observation also confirmed by 1-8 anilinonaphtalene sulphonic acid binding studies. We have also investigated the occurrence of substrate-induced changes in the protein tertiary structure by fluorescence techniques. In particular, eicosatetraynoic acid (ETYA), an irreversible inhibitor of lipoxygenase, has been used to mimic the effect of substrate binding. We demonstrated that mini-Lox is indeed characterized by much larger conformational changes than those occurring in the native Lox-1 upon binding of ETYA. All these findings strongly support the hypothesis that the larger hydration of mini-Lox renders this molecule more flexible and therefore less stable, that on the other hand is probably causing its higher catalytic efficiency.
\end{abstract}

Keywords: Limited proteolysis, intrinsic fluorescence, conformational changes, ANS binding

\section{Abbreviations}

Lox, lipoxygenase; $\mathrm{GdHCl}$, guanidinium hydrochloride; $\mathrm{CD}$, circular dichroism; ETYA, eicosatetraynoic acid; ANS, 1-8 anilinonaphthalene sulfonic acid.

\footnotetext{
${ }^{1}$ These authors contributed equally to this work.

*Corresponding author. Tel.: +390861 266875; Fax: +39 0861 412583; E-mail: Maccarrone@ vet.unite.it.
} 


\section{Introduction}

Lipoxygenases (Loxs) are monomeric enzymes involved in the metabolism of polyunsaturated fatty acids by an iron-mediated reaction. Widespread in animals and plants, the key function of Loxs in the metabolism of fatty acids has been widely demonstrated, as well as their involvement in several human diseases [1]. These features have stimulated a growing scientific interest about both functional and structural aspects of Loxs. In particular, soybean lipoxygenase-1 (Lox-1) has been studied in detail as a prototype for the structural and functional properties of lipoxygenases [2]. Lox-1 is composed of two domains: a $30 \mathrm{kDa}$ N-terminal domain and a $60 \mathrm{kDa}$ C-terminal domain containing the iron-binding site and the hydrophobic cavity housing the substrate. The function of the $\mathrm{N}$-terminal $\beta$-barrel domain has been elusive for several years, for Lox-1 as well as for other Lox iso-zymes [2]. Recently, the N-terminal domain has been shown to be essential for calcium binding and activation of 5-lipoxygenase activity [3] and for nuclear membrane translocation of this Lox isoform [4,5]. Equilibrium unfolding experiments on Lox-1 have shown that the $\mathrm{C}$-terminal domain is less stable than the $\mathrm{N}$-terminal domain [6]. The latter is more resistant to denaturation and appears to retain most of its $\beta$-barrel secondary structure motif even at high urea concentration, suggesting that this portion of the protein might be important for the overall stability. In a previous study limited proteolytic cleavage of Lox-1 generated a 60-kDa fragment (termed "mini-Lox"), almost overlapping the C-terminal domain. It has been shown that the trimmed enzyme is still folded, and displays a higher binding affinity than Lox-1 for artificial membranes, attributable to the enhanced surface hydrophobicity exposed by removing the $30 \mathrm{kDa} \mathrm{N}$-terminal fragment [7]. Interestingly, the conclusions drawn from these studies on the effect of the $\mathrm{N}$-terminal domain removal have been recently extended to the activity and membrane binding ability of the reticulocyte-type 15lipoxygenase [8].

In this paper we have investigated the structural features of mini-Lox, as revealed by circular dichroism, 1-8 anilinonaphthalene sulfonic acid (ANS) binding and steady-state fluorescence. The stability of mini-Lox using chemical equilibrium unfolding measurements and denaturation by hydrostatic pressure has also be studied. The results demonstrate that the removal of the $\mathrm{N}$-terminal domain makes the mini-Lox easily unfoldable by both guanidinium hydrochloride $(\mathrm{GdHCl})$ and pressure. These new data confirm that mini-Lox is more hydrated and suggest a structural role for the N-terminal domain, as recently proposed [9]. Furthermore, ANS binding experiments provide new evidence that the active site is more accessible in mini-Lox than in Lox-1. In order to study the possible protein conformational changes associated to substrate binding, we have also used an irreversible inhibitor of LOXs (eicosatetraynoic acid), demonstrating that the mini-Lox flexibility is much larger than that of Lox-1. All together, these results provide a new structural rationale, which might explain the functional properties of mini-Lox compared to those of native Lox-1.

\section{Experimental procedures}

\subsection{Materials and enzymes}

Linoleic (9,12-octadecadienoic) acid and 5,8,11,14-eicosatetraynoic acid (ETYA) were purchased from Sigma Chemical Co (St. Louis, USA). Ultrapure guanidinium hydrochloride and ANS were purchased from US Biochemical Corp. and Molecular Probes Inc., respectively. Lox-1 (linoleate:oxygen oxidoreductase, EC 1.13.11.12; Lox-1) was purified from soybean (Glycine max [L.] Merrill, Williams) 
seeds as reported [10], while mini-Lox was obtained by tryptic digestion of Lox-1 as described elsewhere [7]. Dioxygenase activity of mini-Lox in $100 \mathrm{mM}$ sodium borate buffer (pH 9.0) was assayed spectrophotometrically at $25^{\circ} \mathrm{C}$, by recording the formation of conjugated hydroperoxides from linoleic acid at $234 \mathrm{~nm}$, using a Perkin Elmer Lambda-18 spectrophotometer [11].

\subsection{Spectroscopic assay}

CD spectra were recorded on a Jasco-710 spectropolarimeter, at $20^{\circ} \mathrm{C}$, using a $0.1 \mathrm{~cm}$ and $1.0 \mathrm{~cm}$ quartz cuvette in the peptidic and visible region, respectively. Steady-state fluorescence spectra upon excitation at 280 or $293 \mathrm{~nm}$, have been collected using a photon counting spectrofluorometer (ISS, Model K2, USA). ANS binding was studied measuring the fluorescence emission spectra (from 420 to $550 \mathrm{~nm})$ of the probe, using a $(350 \pm 2) \mathrm{nm}$ excitation wavelength. High-pressure measurements were performed with the same instrument, using the high-pressure ISS cell, equipped with an external bath circulator [9].

\subsection{Equilibrium unfolding measurements}

Protein denaturation was obtained incubating the protein with different amounts of $\mathrm{GdHCl}$ for $12 \mathrm{~h}$ at $4^{\circ} \mathrm{C}$ (final protein concentration $\approx 1.5 \times 10^{-6} \mathrm{M}$ ). Refolding of fully unfolded mini-Lox samples was achieved by diluting the denaturant concentration with buffer. The analysis of the fluorescence unfolding transition was performed as described elsewhere [12], according to a double step denaturation pathway following the scheme:

$$
\mathrm{N} \stackrel{K_{1}}{\longleftrightarrow} \mathrm{I} \stackrel{K_{2}}{\longleftrightarrow} \mathrm{U}
$$

In this scheme, $\mathrm{N}, \mathrm{I}$ and $\mathrm{U}$ represent the native, intermediate and unfolded protein species, respectively, while $K_{1}$ and $K_{2}$ are the two equilibrium constants.

The CD unfolding transition was analyzed assuming a two-state equilibrium model between intermediate and unfolded species:

$$
\mathrm{I} \stackrel{K_{2}}{\longleftrightarrow} \mathrm{U}
$$

\section{Results and discussion}

\subsection{Denaturation of mini-Lox}

The effects of chemical denaturants on mini-Lox were analyzed by several spectroscopic techniques. In particular $\mathrm{CD}$ and steady-state fluorescence measurements at different $\mathrm{GdHCl}$ concentration demonstrate that the mini-Lox unfolding transition is rather complex and not compatible with a simple twostate denaturation model (Fig. 1). The best-fit values of the data are consistent with the presence of a stable intermediate species, obtained at $1.5 \mathrm{M} \mathrm{GdHCl}$ and characterized by a very low free energy change $(\Delta G \approx 1.0 \mathrm{kcal} / \mathrm{mol})$. From the data of Fig. 1 it is evident that this state has a native-like secondary structure but it is partially unfolded as revealed by the fluorescence peak position $(\approx 342 \mathrm{~nm})$. It has also been previously demonstrated that at $1.5 \mathrm{M} \mathrm{GdHCl}$ the protein is inactive [9]. All together 


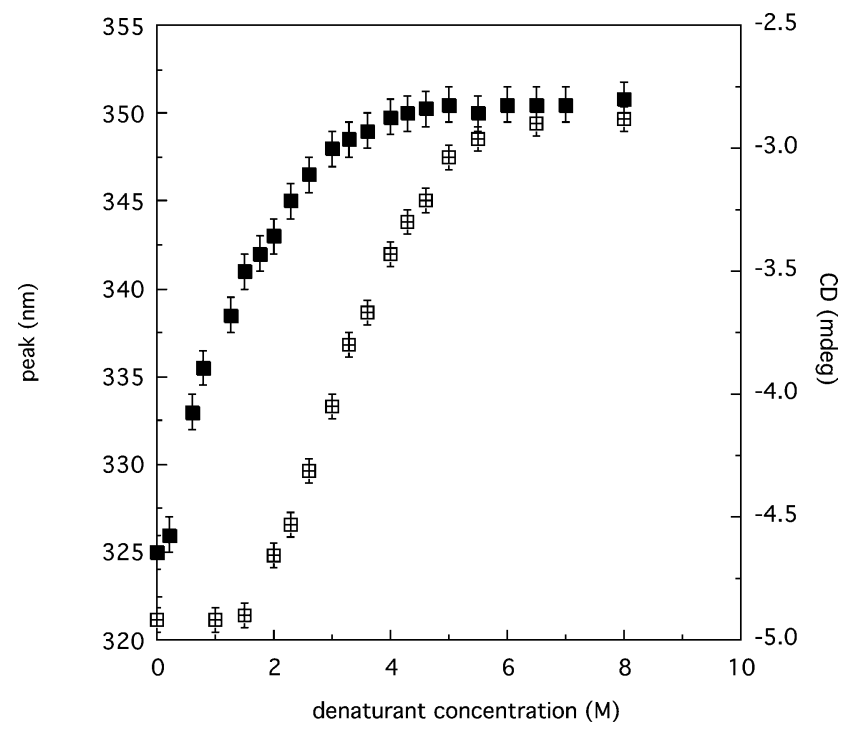

Fig. 1. Dependence of the fluorescence emission maximum (filled symbols) and of circular dichroism at $220 \mathrm{~nm}$ (open symbols) of mini-Lox on $\mathrm{GdHCl}$ concentration.

these characteristics resemble those of the so-called "molten globule" state already found for several other proteins $[13,14]$. In addition, complete analysis of these data revealed that the total free energy change of unfolding from the native to the completely denatured structure (at $6 \mathrm{M} \mathrm{GdHCl}$ ) is rather low $\left(\Delta G_{\mathrm{tot}}^{0} \approx 4.1 \pm 0.3 \mathrm{kcal} / \mathrm{mol}\right)$ especially if compared to that of Lox-1 $\left(\Delta G_{\mathrm{tot}}^{0} \approx 26 \mathrm{kcal} / \mathrm{mol}[6]\right)$. This result suggests a protective role for the $\mathrm{N}$-terminal domain of Lox-1, in keeping with the different hydration of these two molecules [9].

\subsection{The effects of ETYA-binding on mini-Lox}

As already mentioned, a previous study has demonstrated that mini-Lox is more active than Lox-1 [7]. In this context we have investigated which conformational changes were involved in the enzymatic activity and if they were correlated to its lower stability. In particular, the occurrence of substrate-induced changes of the protein tertiary structure has been studied using ETYA, an irreversible inhibitor of lipoxygenase. The analysis of the protein secondary structure through CD spectroscopy revealed that no significant changes were induced by ETYA on either Lox-1 or mini-Lox (data not shown). Different results have been obtained in the aromatic and visible regions (Fig. 2). Lox-1 does not have a significant signal in these regions [15] and the ETYA binding does not influence its CD. Mini-Lox is instead characterized by a very peculiar spectrum clearly structured in individual bands (Fig. 2) confirming the existence of some differences between the two enzymes at the level of the tertiary structure, also near the iron co-factor and the catalytic binding site. Furthermore, in the presence of ETYA the spectrum was less structured, as demonstrated by the flattening of all peaks. The profile of this spectrum suggests that only in this case important changes in the mini-Lox tertiary structure occur when ETYA was bound.

These results have been confirmed by the data of Fig. 3, where the steady-state fluorescence spectra of Lox-1 and mini-Lox have also been reported upon ETYA binding. In both cases the inhibitor quenches the emission intensity in about $30 \mathrm{~min}$, but to different extents. In the case of Lox-1 (Fig. 3a) the fluorescence spectrum does not change its profile, while, when added to mini-Lox (Fig. 3b), the "blue" 


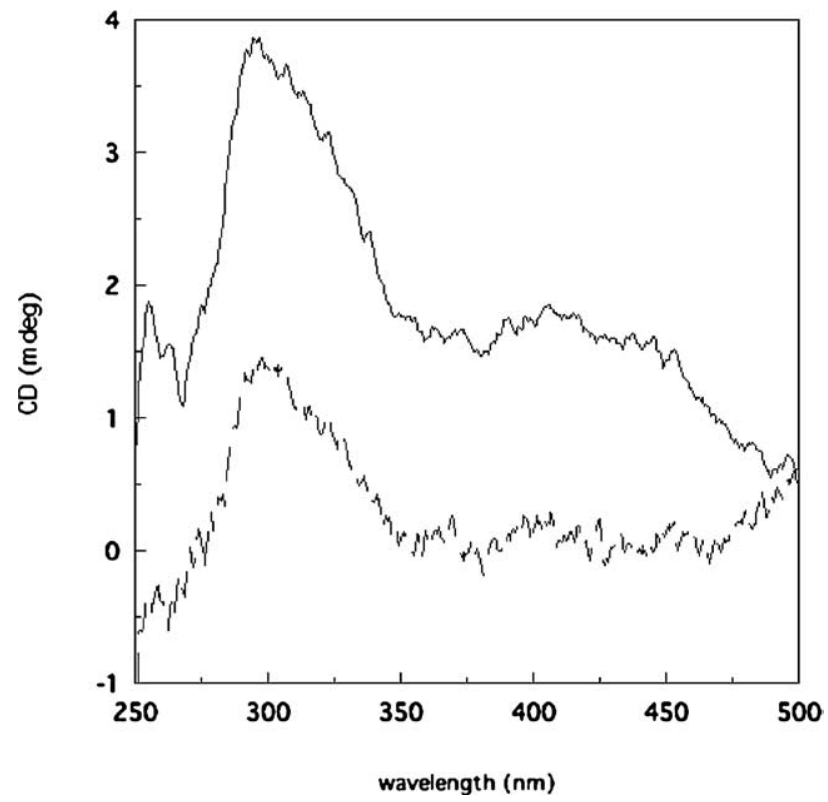

Fig. 2. CD spectrum of mini-Lox alone (solid line) and upon binding of ETYA (dashed line, at a final ratio $1: 20$ ).
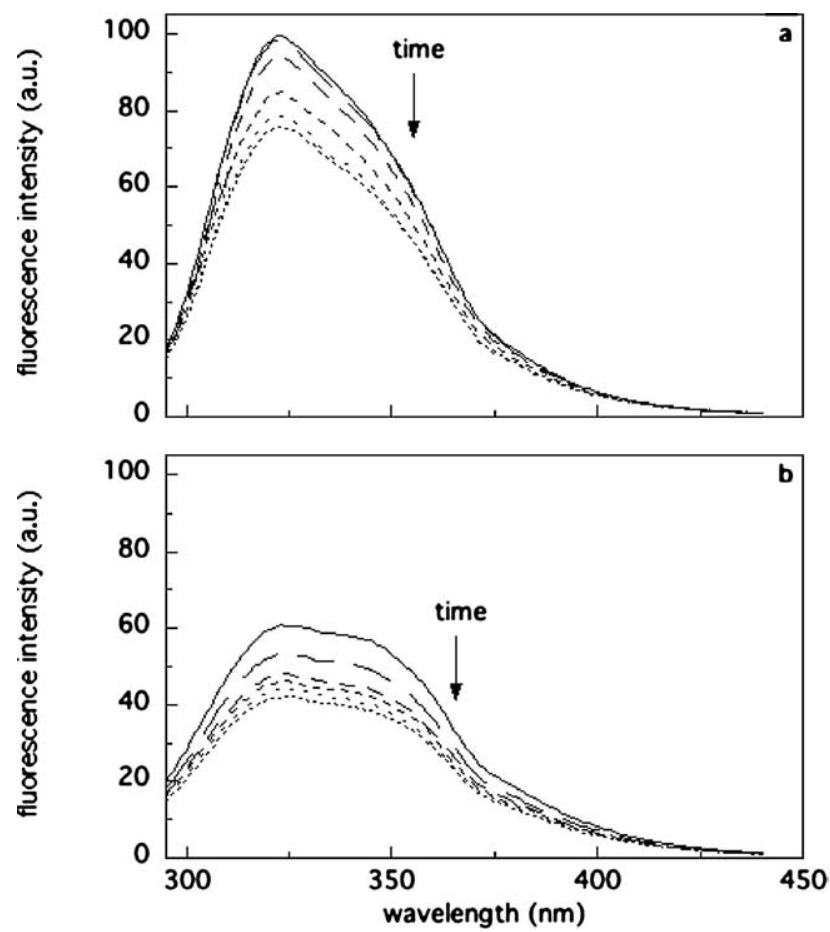

Fig. 3. (a) Steady state emission spectra of Lox-1 alone (solid line) and upon incubation with 20-fold molar excess of ETYA (dotted lines), as a function of time (the spectra are recorded every 5 minutes). (b) Steady state emission spectra of mini-Lox alone (solid line) and upon incubation with 20-fold molar excess of ETYA (dotted lines), as a function of time (the spectra are recorded every 5 minutes). 


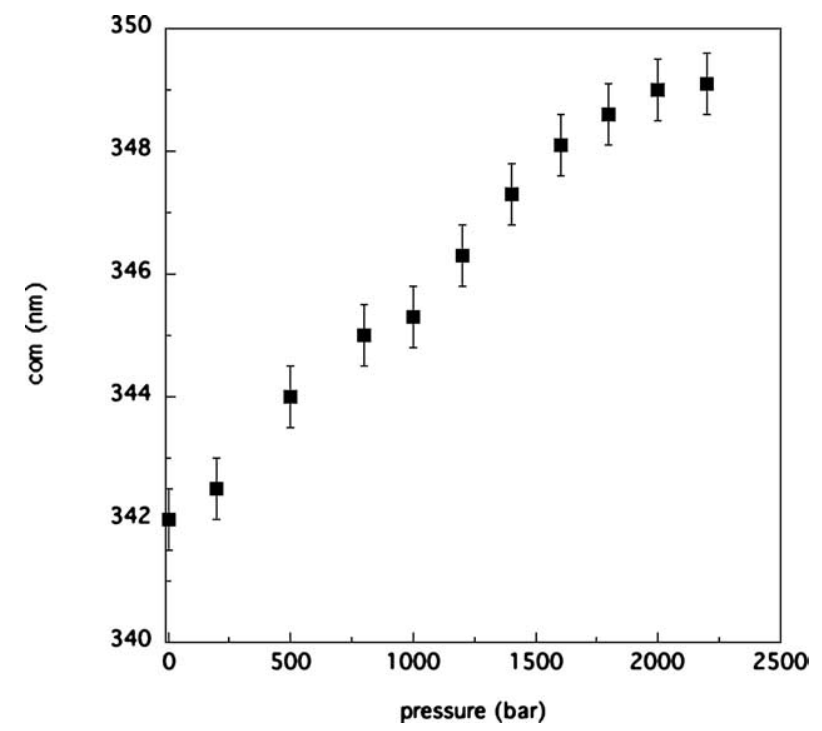

Fig. 4. Dependence of the fluorescence center of mass of mini-Lox bound to ETYA as a function of pressure.

region, i.e. the less hydrated tryptophans of the protein, is preferentially quenched. The slow change of the fluorescence intensity indicates that the quenching mechanism is indirect, probably due to some protein conformational changes. As a matter of fact, previous studies [16-19] have shown that some tryptophylic residues are located in the hydrophobic substrate-binding site and are also essential for the enzymatic activity of Lox-1. Interestingly, it has been also demonstrated [9] that the time-course of these processes is different. The effects of the conformational changes induced by ETYA on mini-Lox stability have been studied, by combining high pressure and fluorescence spectroscopy. High pressure is, in fact, a physical denaturant widely used to study protein stability. It has been previously reported [9] that at 2400 bar mini-Lox is partially unfolded while the effects of high pressure on mini-Lox bound to ETYA are quite different. In particular, the fluorescence spectra are more red-shifted demonstrating that water easily penetrates in the protein core and that at 2400 bar the enzyme is almost completely unfolded (Fig. 4). In the case of Lox-1, ETYA does not have effects on the protein stability (data not shown). One possible explanation for all these different behaviors might be the larger flexibility and degrees of freedom experienced by the mini-Lox molecule. Thus, larger conformational changes might be expected for mini-Lox upon ETYA binding.

\subsection{ANS binding experiments}

ANS is a well-known fluorophore, which increases its fluorescence quantum yield upon binding to hydrophobic regions of proteins. This effect is generally accompanied by a blue-shift in the ANS fluorescence spectrum, which can be detected by evaluating the change in the spectral center of mass. These properties have been largely exploited to check the presence of hydrophobic pockets in proteins through ANS binding. A previous study on Lox-1 has demonstrated that this protein has one binding site for ANS, which does not affect enzyme activity [20]. The reported unfolding measurements and spectroscopic assays of mini-Lox have pointed out that the main structural differences compared to Lox-1 are associated with the solvent accessibility and the tertiary structure. We have therefore studied the binding of ANS to both proteins. The results reported in Fig. 5 demonstrate that the occupancy of the active site 


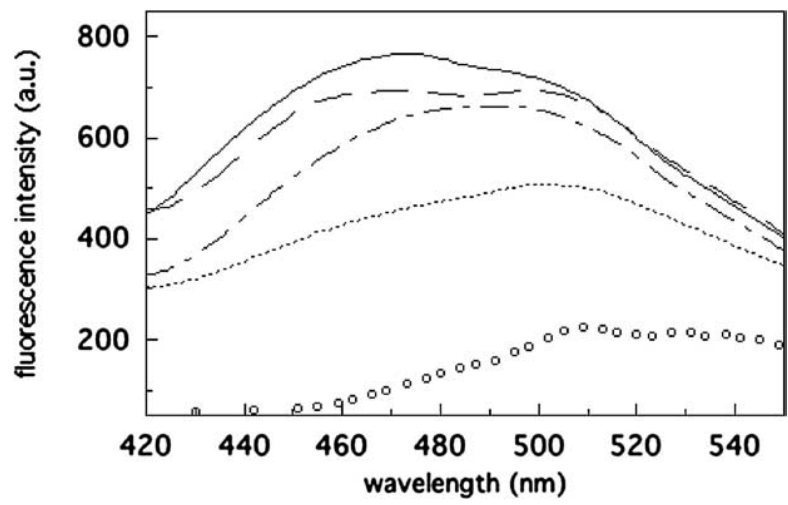

Fig. 5. Fluorescence spectrum of ANS (circles) and of ANS bound to Lox-1 (-) or to mini-Lox (- - -) after 30 min of incubation (at an ANS to protein ratio of $5: 1)$. The measurement was repeated by pre-incubation of Lox $(\cdots)$ or mini-Lox $(-\cdot-)$ with ETYA (20-fold molar excess).

by ETYA reduced the ANS fluorescence intensity only when the probe was bound to mini-Lox, suggesting that the two molecules compete for the mini-Lox catalytic site. In the case of Lox-1 no significant changes in ANS intensity and peak position were recorded (Fig. 5).

\subsection{Conclusions}

In this study we attempted to explain which possible correlation might exist between biological and structural features of mini-Lox. We have shown that the removal of the $30 \mathrm{kDa}$ fragment produces a more hydrated enzyme, which displays an increased affinity for hydrophobic probes like ANS. Mini-Lox appears to undergo larger conformational changes than Lox-1 upon ETYA and ANS binding, displaying also greater sensitivity to pressure- and $\mathrm{GdHCl}$-induced unfolding. It could be argued that a larger structural flexibility due to the partial loosening of the tertiary structure is the main reason for the enhanced activity. In particular, the ability to bind ANS in the active site is a direct evidence that mini-Lox gives the substrates an easier access to the hydrophobic cavity, where the catalysis takes place. In this context, the hypothesis that the N-terminal domain of Lox-1 could act as a built-in inhibitor of the enzymatic activity [7] takes ground. The N-terminal fragment could also make the native enzyme less prone to associate with hydrophilic molecules, and more selective towards hydrophobic molecules like its natural lipid substrates.

\section{Acknowledgements}

We wish to thank Dr. Maria Luisa Salucci (University of L'Aquila, Italy) for helpful assistance in mini-Lox preparation.

This study was supported in part by grants from Istituto Superiore di Sanità (IV AIDS project), Agenzia Spaziale Italiana (contract I/R/098/00), Ministero Politiche Agricole e Forestali (project FORMINNOVA 2002), and Ministero dell'Istruzione, dell'Universita' e della Ricerca (project COFIN 2002), Rome. 


\section{References}

[1] H. Kühn and S. Borngraber, Adv. Exp. Med. Biol. 447 (1999), 5-28.

[2] A.R. Brash, J. Biol. Chem. 274 (1999), 23679-23682.

[3] T. Hammarberg, P. Provost, B. Persson and O. Radmark, J. Biol. Chem. 275 (2000), 38787-38793.

[4] X.S. Chen and C.D. Funk, J. Biol. Chem. 276 (2001), 811-818.

[5] S.M. Jones, M. Luo, A.M. Healy, M. Peters-Golden and T.G. Brock, J. Biol. Chem. 277 (2002), 38550-38556.

[6] E. Sudharshan and A.G. Appu Rao, J. Biol. Chem. 274 (1999), 35351-35358.

[7] M. Maccarrone, M.L. Salucci, G. van Zadelhoff, F. Malatesta, G. Veldink, J.F.G. Vliegenthart and A. Finazzi-Agrò, Biochemistry 40 (2001), 6819-6827.

[8] M. Walther, M. Anton, M. Wiedmann, R. Fletterick and H. Kühn, J. Biol. Chem. 277 (2002), 27360-27366.

[9] A. Di Venere, M.L. Salucci, G. van Zadelhoff, G. Veldink, G. Mei, N. Rosato, A. Finazzi-Agrò and M. Maccarrone, J. Biol. Chem. 278 (2003), 18281-18288.

[10] A. Finazzi-Agrò, L. Avigliano, G.A. Veldink, J.F.G. Vliegenthart and J. Boldingh, Biochim. Biophys. Acta 326 (1973), 462-470.

[11] M.J. Schilstra, G.A. Veldink and J.F.G. Vliegenthart, Biochemistry 33 (1994), 3974-3979.

[12] A. Di Venere, A. Rossi, N. Rosato, F. De Matteis, A. Finazzi-Agrò and G. Mei, J. Biol. Chem. 275 (2000), 3915-3921.

[13] K. Kuwajima, Proteins 6 (1989), 87-103.

[14] O.B. Ptitsyn, R.H. Pain, G.V. Semisotnov, E. Zerovnik and O.I. Razgulyaev, FEBS Lett. 262 (1990), 20-24.

[15] L.J. Spaapen, G.A. Veldink, T.J. Liefkens, J.F. Vliegenthart and C.M. Kay, Biochim. Biophys. Acta 30 (1979), 301-311.

[16] A. Finazzi-Agrò, L. Avigliano, M.R. Egmond, G.A. Veldink and F.G. Vliegenthart, FEBS Lett. 52 (1975), 73-76.

[17] B.P. Klein, B.S. Cohen, S. Grossman, D. King, H. Malovany and A. Pinsky, Phytochemistry 24 (1985), 1903-1906.

[18] J.C. Boyington, B.J. Gaffney and L.M. Amzel, Science 260 (1993), 1482-1486.

[19] E. Sudharshan, S. Srinivasulu and A.G. Appu Rao, Biochim. Biophys. Acta 1480 (2000), 13-22.

[20] E. Sudharshan and A.G. Appu Rao, FEBS Lett. 406 (1997), 184-188. 


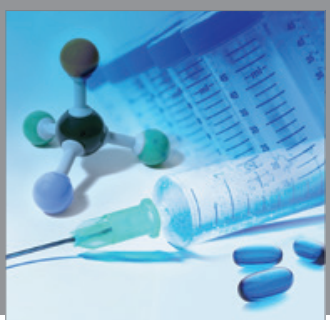

International Journal of

Medicinal Chemistry

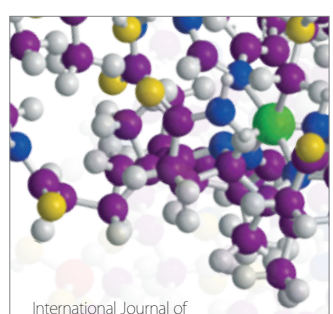

Carbohydrate Chemistry

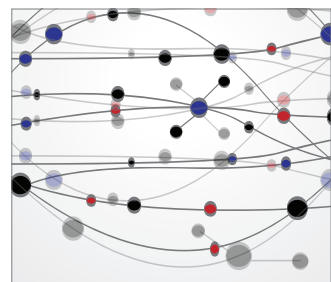

The Scientific World Journal
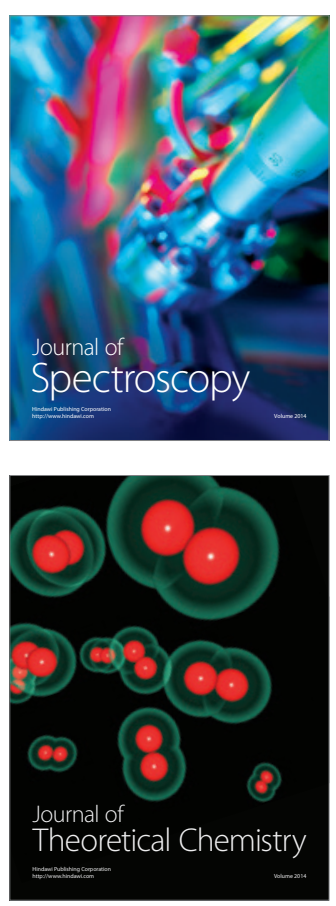
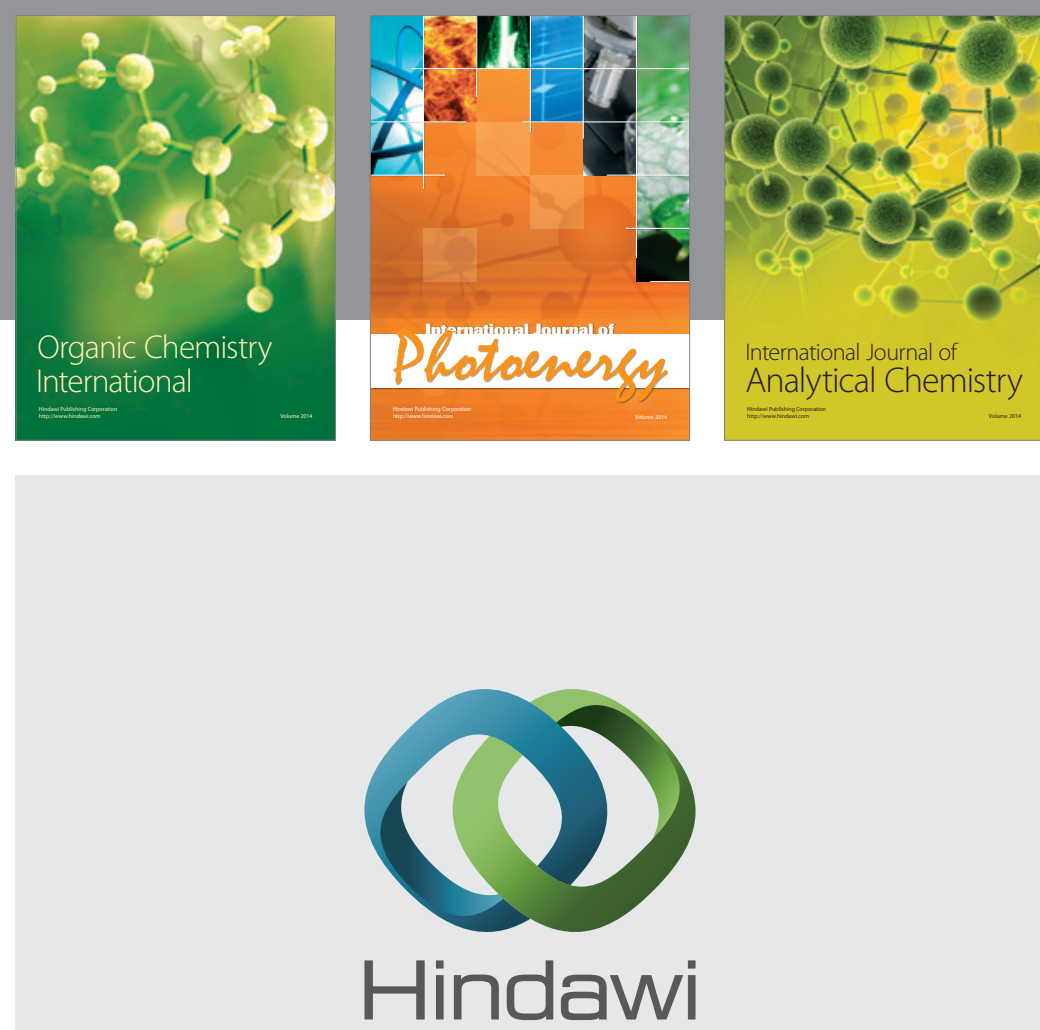

Submit your manuscripts at

http://www.hindawi.com
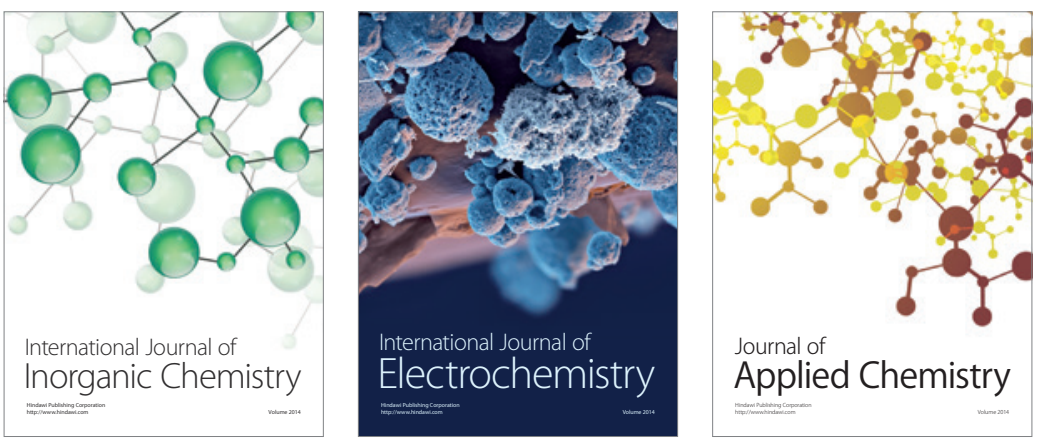

Journal of

Applied Chemistry
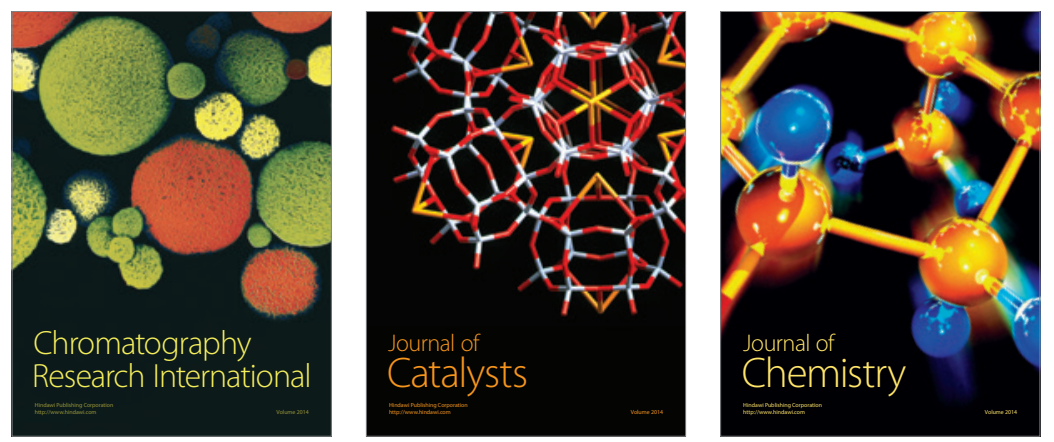
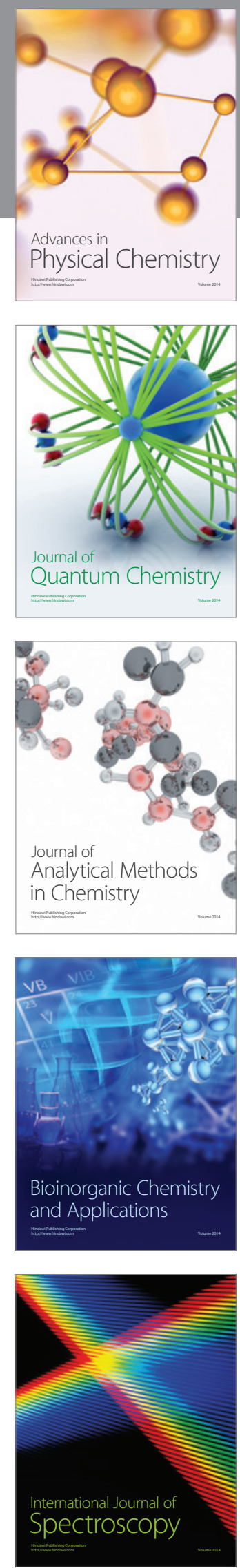